\title{
LA HETERÓCLISIS EN HETITA
}

Hittite presents two types of alternance:

The type $-r / n-$, which is inherited and relatively productive, forms nouns within well-defined semantic fields: temporal designations: mehur mehunaš; body-parts: kuttar kuttanaš; fluids: watar wetenaš, and basic natural elements: pahhur pahbuenaš.

The type $-r / \emptyset$ appears only in the suffix -war/-waš which forms abstract nouns like taruppuwar taruppuwaš 'meeting'. It is a development exclusive of Hittite. There are three suffixes inflected by the alternance $-r / n-;-(a)$ tar $/-(a) n n a s ̌<<$ -(a)tnaš; -eššar/-ešnaš; -war/-unaš.

The great development of these suffixes - an exclusive feature of Hittiteseems to be a peculiar innovation of this language, destined to integrate certain stems in its flexion:

The suffix -tar/-nnaš, supposedly the inheritor of an I.E. suffix *-ter/-tn-, is used again in order to integrate the ${ }^{*}-\mathrm{eH}_{2} /-\mathrm{H}_{2}$ stems, which formed abstract nouns already from the I.E. and did not present any other productive type in Hittite. This would be the suffix -atar/-annaš.

The suffix -eššar/-ešnaš integrates the *-es/-os I.E. nouns in the Hittite nominal system, which formed abstract nouns already in I.E.; this suffix does not appear in other inflected type in Hittite.

The suffix -war/-unaš, on the contrary, is not productive and can create designations of parts of animal bodies and abstract nouns as well.

0 . Cuando el estudioso se acerca el tema de la heteróclisis nominal en las lenguas ides., encuentra que los manuales repiten casi uniformemente la clasificación que en su día presentó Benveniste' ${ }^{1}$. Cuando se acerca a la heteróclisis en hetita, el estudioso comprueba que esta clasificación no se ajusta en varios puntos a los hechos reales de la lengua ${ }^{2}$.

En líneas generales, los temas alternantes que se dan en het. son el tipo $-r / n$ - y un tipo exclusivo del het., llamado por Villar ${ }^{3}$ en $-r / \emptyset$-, este último sólo en un sufijo.

1 E. Benveniste, Origines de la formation des noms en indoeuropéen, París 1935.

2 Efectivamente, no existen en het. los tipos en $-i / n,-\emptyset / n-y-l / n-$, que serían muy arcaicos, según Benveniste, y habrian jugado un papel muy importante en la primitiva flexión del ide.

${ }^{3}$ F. Villar, Origen de la flexión nominal indoeuropea, Madrid 1974, p. 92. 
También hay que distinguir entre temas simples de alternancia $-r / n$ y sufijos flexionados mediante heteróclisis.

No tomaremos en cuenta otros conceptos de heteróclisis ${ }^{4}$, ni admitiremos la existencia en het. de un tipo de alternancia en $-a / n-5$.

1. En los temas heteróclitos simples de alternancia $-r / n$ - nos encontramos con una falta de sistematicidad en el empleo del vocalismo de los sufijos ${ }^{6}$. En het. la ley de equilibrio silábico ${ }^{7}$ que determina la obligatoriedad de la relación raíz en grado pleno / sufijo en grado cero y viceversa está ya relajada ${ }^{8}$ y nos encontramos incluso con alomorfos de una misma palabra que responden precisamente a esa falta de aplicación estricta del mencionado principio: pahhur/pahhuwar, mehuni/mehueni.

Otro aspecto que conviene señalar es que la solución *-r en hetita no es uniforme; hay vocalizaciones de timbre $a$ - y vocalizaciones de timbre $u$-. El tratamiento de timbre $a$ - es el más frecuente: ešhar 'sangre'. En cambio $u$ - aparece siempre tras $g / k$-, que esconde una antigua labiovelar: zamankur 'barba', y tras laringal $h$-: sehur 'orina' 9 .

4 Los numerosos paradigmas a los que E. H. Sturtevant, A Comparative Grammar of the Hittite Language, New Haven 1964, pp. 94-9, denomina formas heteróclitas suelen ser desajustes de número: e. d. formas de plural coincidentes con las de singular. En realidad el het. no ha diferenciado estrictamente todos los casos del plural (v. Villar, o. c., pp. 61-5). También denomina Sturtevant heteróclitos a nuevas formaciones temáticas a partir de la forma de genitivo, tipo N.sg. ki-eš-ši-ra-a-aš, Ac.sg. ki-iššse-ra-an, Ac.pl. ki-iš-še-ru-uš sobre el N.sg. ki-eš-šar 'mano' (Sturtevant, o. c., p. 96). Cualquier palabra está expuesta en realidad a que su genitivo sea interpretado como nominativo y reciba un nuevo acusativo en -an (Villar, o. c., pp. 73-8).

${ }^{5}$ Serian temas en -a/n-palabras del tipo N. haraš, G. haranaš c., N. memiyaš, G. memiyanaš c., en donde, según Sturtevant (o. c., p. 95), el nominativo funciona como un tema en - $a$ (e. d. temático) y los demás casos como temas en - $n$. Para Benveniste (o. c., p. 24) seria un tipo especial de heteróclisis en el que el nominativo-acusativo es el tema puro frente a los casos oblicuos con $-n$, y lo compara con el tipo ai. ás àsnás, etc. Preferimos seguir la interpretación de Villar (o. c., pp. 81-5), mucho más ajustada a los hechos reales del hetita. Junto a las formas ya mencionadas aparecen otras sin alargamiento en $-n$ : N. haraš, A.sg. baran; N. memiyaš, A.sg. memiyan, G.D.pl. memiyaš, etc., que son las realmente antiguas. Las formas en $-n$ son secundarias, refecciones del het. para superar la identidad entre el nominativo y el genitivo de los nombres temáticos. Los casos secundarios con alargamiento en $-n$ se derivan a partir del A.sg.: de haran G. haranaš y a partir de éste un nuevo acusativo: haranan.

6 J. Schmidt, Die Pluralbildungen der indogermanischen Neutra, Weimar 1889 . p. 172 ss.

7 Benveniste, o. c., p. 26.

${ }^{8}$ Villar, o. c., p. 90.

${ }^{9}$ F. R. Adrados, Estudios sobre las sonantes y laringales indoeuropeas, Madrid 1973 , p. 53, atribuye estas últimas soluciones a la serie de laringales con apéndice velar šehur $<{ }^{*} \mathrm{seH}_{1}{ }^{*}$. 
1.1. Son heteróclitos en -ar/-anaš once nombres. En ellos la grafia -ar es ambigua y puede representar tanto ${ }^{*}-r$ como ${ }^{*}$-or.

1.1.1. N.A. ešḩar, eššar, G. ešhanaš, išhanaš, ešnaš, D.L. ešhani, Ab. ešhanaz(a), I. ešhanta 'sangre', cf. el compuesto luv. sobre tema en $-r$ : ašharnumanzi ${ }^{10}$ y sobre el tema en - $n$ : ašhanuwantiš N.sg.c. 'mancha de sangre'; quizá se puedan incluir aquí luv. jerogl. átar c. (tema en -a), el Ac.sg.c. átar(a)n y la forma oscura ?334-tari" ${ }^{\prime \prime}$. En palaíta la palabra para 'sangre' se presenta siempre en el compuesto hinapešhur, con vocalización $-u$ tras laringal; tiene un locativo de lectura controvertida, pero que quizá testimonie un tema en -n: (-)e-eš-ha-n[a(-); el lic. esd- podría ser la evolución de *ašhata(-r $)^{12}$.

Conservan el tema en - $n$ : ešhanant-, išhanant- 'sangre', ešhanuwant'ensangrentado', išhanittaratar 'parentesco de sangre', išhanittalla- 'pariente consanguíneo'. En cambio, se forman sobre el tema en -r: ešharnu- ešharnumai- išharnumai- 'ensangrentar', ešharwant- išharwant- 'rojo (sangre)', ešharwiya- (?) 'sangrar', ešharwil- 'hemorragia' 13 .

Se compara con ai. áş̧k asnás, toc. A ysär B yasar, lit. asins, arm. ariwn, gr. kap, lat. arc. assyr (Fest. 15, 13), corregido por las glosas en aser asser.

1.1.2. N.A. haršar, G. haršanaš, D.L. harašni, haršani, Ab. haršanaza 'cabeza' es comparado habitualmente con el ai. siras sírsnás aunque con reparos, debido al problema fonético que implica el resultado het. $h$ - de ${ }^{*} k-{ }^{14}$.

Sin embargo, A. J. Nussbaum ${ }^{15}$ piensa que haršar es un desarrollo propio del hetita y que no tiene ninguna relación etimológica directa con la raíz que da lugar a ai. śíras, gr. kápa, sino que está relacionado con het. ${ }^{D U G}$ harši- 'pithos', por lo que sería una evolución similar a la de lat. testa > it. testa 'cabeza'.

${ }^{10}$ H. Kronasser, Etymologie der hethitischen Sprachen (EHS), Wiesbaden, p. 321; R. Stefanini, "Itt. ešhar (=sangue): Problemi formali ed etimologici», AGI 43 , pp. 18-41.

$"$ P. Meriggi, Manuale di Eteo Geroglifico, Roma 1966, p. 43 ss.

12 O. Carruba, Das Palaische, Texte, Grammatik, Lexicon, Wiesbaden 1970, p. 53.

13 J. Tischler, Hethitisches Etymologisches Glossar (HEG), 1977, p. 115.

14 Kronasser, EHS, p. 281; A. Bernabé, «Designaciones de la cabeza en las lenguas indoeuropeas", Athlon, Hom. F. R. Adrados, Madrid 1984, p. 101 s.; se explica habitualmente como un fenómeno de fonética expresiva, o quizá por analogía con otra palabra para 'cabeza': halanta-. De todos modos, es posible que la caída de la laringal existente en esa raiz: ${ }^{*} k e r H-$, o ${ }^{*} k e r H^{*}$ - como postula Bernabé, haya presionado para dar lugar a esta solución (v. M. Peters, Untersuchungen zur Vertretung der indogermanischen Laryngalen im Griechischen, Viena 1980, p. 230).

is A. J. Nussbaum, Head and Horn in Indo-European, Berlin-Nueva York 1986, p. 22. 
Este nombre se tematiza sobre el tema en - $r$ en haršara- o extiende el tema en $-n$ a toda la flexión: haršana- n., haršana- c. ${ }^{16}$.

1.1.3. N.A. kuttar, D.L. kuttani, I. kuttanit 'parte bajo el cuello y sobre el pecho entre los hombros'. Parece muy probable la comparación con lat. guttur (m. y n.), nal. koden 'papada', nal. dial. Koder Köderl 'papada, garganta' ${ }^{17}$. Puede que nos encontremos ante un deverbativo sobre el verbo ide. ${ }^{*} g \bar{e} u$ - gi $\overline{\text { - }}$ 'torcer, retorcer' ${ }^{18}$; het. kuttar sería un abstracto antiguo en -tar de un comportamiento especial ya que no hace su genitivo en -tn-aš > -nnaš; quizá los hablantes ya no establecían una relación etimológica entre el abstracto y su palabra base, v. infra uttar $(\S 1.1 .10)$.

1.1.4. N.A. lammar, G. lamnaš, D.L. lamni 'hora, momento' adv. 'inmediatamente', disimilación de * nomor, cf. lat. numero 'aprisa' ${ }^{19}$.

1.1.5. N.A. mukar, Ab. muknaza 'un instrumento musical' ${ }^{20}$.

1.1.6. N.A. pattar, G. pattanaš 'ala' se relaciona con el verbo piddai'volar'; el tema en $-r$ se conserva en el compuesto pattarpalhi- 'de ala ancha (?)'; formaciones en $-r$ o $-n$ sobre este tema son gr. птعрóv, ai. pátram, lat. penna, aisl. fjodr, airl. én ${ }^{21}$.

1.1.7. N.A. ${ }^{\text {GI }}$ pattar, D.L. paddani, Ab. paddana(z), I. pattanit 'cesto ritual', cf. gr. пaтávi, lat. patera, lic. parara 'cesto, armario' ${ }^{22}$. A. Götze $^{23}$ da la variante ${ }^{\text {GIS }}$ paddur (cf. infra $\S 1.2 .2$ ).

1.1.8. N.A. šakkar zakkar, G. šaknaš 'excremento' se compara habi-

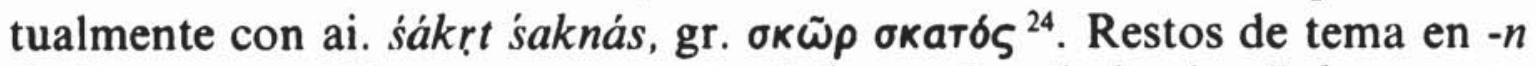
de los casos oblicuos aparecen también en los derivados šakn-uwant-, šak[n]-umar, šakn-iya-.

16 Tischler, $H E G$, p. 184.

17 J. Friedrich, Hethitisches Wörterbuch $(\mathrm{HW}), 2$. Ergänzungsheft, Heidelberg 1961 , p. 16; Kronasser, EHS, pp. 279-82; A. Kammenhuber, «Zur Genese der hethitischen -r-/-n- Heteroklitika», Corolla Linguistica: Festschrift F. Sommer, Wiesbaden 1955, pp. 97-106.

18 J. Pokorny, Indogermanisches Etymologisches Wörterbuch (IEW), Berna-Munich 1959 , p. 393.

19 Friedrich, Hethitisches Elementarbuch, Heidelberg 1974; HW, Erg. 1957, p. 12; Kronasser, EHS, p. 282; H. G. Güterbock - H. A. Hoffner, The Hittite Dictionary of the Oriental Institute of the University of Chicago $(L-M A), 1980$, p. 36 ss.

${ }^{20}$ Kronasser, EHS, p. 282.

${ }^{21}$ Friedrich, $H W$, Erg. 1952, p. 116; Kronasser, EHS, p. 284; P. Brossman, "The Hittite Genders of Cognates of PIE Neuters», JIES 6, 1978, p. 95.

${ }_{22}$ Friedrich, $H W$, Erg. 1952, p. 116, Erg. 1957, p. $15 \mathrm{~s}$.

${ }^{23}$ A Götze, «Review of Keilschrifttexte aus Boghasköy, by H. Güterbock and $\mathrm{H}$. Otten", Journal of Cuneiform Studies 16, 1962, p. 30.

${ }^{24}$ Friedrich, $H W$, Erg. 1957 , p. 22; Benveniste, o. c., p. 9; "Etudes hittites et indo-européennes", BSL 38, 1954, p. $35 \mathrm{~s}$. 
1.1.9. N.A. šuppiwašhar, G. šuppiwašhanaš 'cebolla', sin etimología clara, pertenece formalmente a este grupo y es interpretado por Kronasser ${ }^{25}$ como un compuesto de šuppi- 'limpio' y -wašhar (?). Es un calco del sum. šum-šikil 'cebolla' (e. d. 'ajo limpio').

1.1.10. N.A. uttar, G. uddanaš, D.L. uddani, Ab. uddanaz(a), I. uddanit, uddanta, N.A.pl. uddar 'palabra' ${ }^{26}$; R. Gusmani ${ }^{27}$ lo asocia a la raíz *wed-, como ai. vádati 'hablar', aesl. vaditi 'acusar', gr. aúón 'voz'. Bernabé ${ }^{28}$ lo incluye en el grupo de Pokorny $\left(I E W\right.$, p. 135) * wek" ${ }^{\psi_{-}}$"hablar', ai. vákti, gr. elnov, lat. uox; procedería de una asimilación * $u k^{\psi}$ tar $>{ }^{*}$ uktar $>$ uttar. Sería, por tanto, un abstracto verbal en -tar, pero con un comportamiento especial, ya que no tiene un genitivo regular en -nnaš < -tnaš (cf. supra kuttar § 1.1.3). También aquí habría desconexión etimológica.

1.1.11. N.A. watar, wedar, uwitar; G. wetenaš; D.L. weteni; Ab. wetenaz $(a)$; I. wetenit, wedanda; N.A.pl. widar, con la variante gráfica N.A. $A$-tar, D.L. $A$-ni, I. A-etenit; hay también una grafia tardía N.A. witar, Ab. witenanza, D.L.pl. wetenaš ${ }^{29}$; conservan el tema en $-r$ : wattaru- 'manantial, fuente', wattariya- 'manar', wattatra- 'fuente'. Restos de un nombre-raiz muy antiguo los tenemos en el D.L. witi, Ab. witaz ${ }^{30}$. Estaría directamente emparentado con luv. Ac.pl.c. widanza y con los

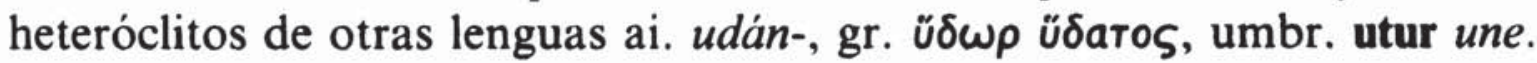

1.2. Con vocalización $u$ - de $-r$ tras velar grafia $g / k$ o tras laringal $h$ hay cinco nombres. Benveniste ${ }^{31}$ piensa que se trata de un alargamiento $-r / n$ - de un tema en $-u$. Según Adrados ${ }^{32}$ y Bernabé ${ }^{33}$ se trata de la vocalización $-u$ del apéndice velar de la labiovelar o de la laringal con apéndice velar ${ }^{*}-H^{\mu}$. Hay una sola excepción, quizá aparente: ${ }^{G I S}$ paddur.

Es importante señalar, pues, que estas formas no son el grado cero del sufijo ${ }^{*}$-wer/-un-, como piensa $\mathrm{H}$. Eichner ${ }^{34}$, sino heteróclitos en $-r$ / $n$ - con vocalización condicionada $-u r<-r$.

25 Kronasser, EHS, p. 285.

26 De etimología desconocida para Kronasser, EHS, p. 284.

27 R. Gusmani, Il lessico ittito, Nápoles 1968, p. 46.

${ }_{28}$ Bernabé, Hetita y Laringales Indoeuropeas, Tesis doctoral de la Univ. Complutense (inédita), 1973, p. 181.

${ }^{29}$ Friedrich, $H W$, Erg. 1961, p. 27; Kronasser, EHS, p. 282.

30 Nussbaum, o. c., p. 203.

31 Benveniste, o. c., p. 37.

32 Adrados, o. c., p. 53.

${ }^{33}$ Bernabé, o. c., p. 571. 107.

${ }^{14}$ H. Eichner, "Die Etymologie von hethitische mehur", MSS 31, 1973, pp. 53- 
1.2.1. N.A. mehur; G. mehunaš; D.L. mehuni, mehueni 'tiempo' ${ }^{35}$.

1.2.2. Si aceptamos que el N.A. GISpaddur, D.L. GISpadduni 'cesto' es variante de ${ }^{G l}$ pattar, habrá que admitir una posibilidad de vocalización ${ }^{*}-r>-u r$ analógica de los casos que estamos tratando. Bernabé ${ }^{36}$, no obstante, postula una laringal previa: ${ }^{*}$ pot $H^{\text {wor }}>{ }^{*}$ pathur $>$ paddur, pattar, con lo que une ambos resultados.

1.2.3. También incluimos en este apartado el nombre N.A. pahhur pahhuwar, G. pahhuenaš, D.L. pahhuni pahhueni, Ab. pahhunaz(a) pahhuenaz(a), I. pahhuenit 'fuego', en el que se da una alternancia entre -ur grado cero /-war grado pleno. Ahora bien, se cuestiona la antigüedad de la formación en grado pleno pahhuwar pahhuenaš. Parece evidente que ambas son formaciones coetáneas desde el momento en que aparecen en la misma tablilla ${ }^{37}$.

Sobre el tema en -r se formará pahhurul 'herramienta de purificar con fuego' y sobre el tema en - $n$ : pahhu(i)nalli- 'brasero' ${ }^{38}$.

Se compara con gr. nũp, umbr. pir, arm. hur, toc. A poräm, aaa. fiur; el tema en $-n$ aparece en got. fon.

1.2.4. N.A. pankur, G. pankunaš, I. pankunit 'parentesco, estirpe' ${ }^{39}$. Bernabé ${ }^{40}$ asimila este nombre a panku- 'todo' a partir de un significado de pankur como 'totalidad' y de ahi 'abundancia, cantidad, riqueza' $y$, frecuentemente y en relación con este último, 'leche'. De ahi la idea de 'parentesco' como 'hermandad de leche'.

1.2.5. N.A. šehur, G. šehunaš, D.L. šehuni 'suciedad, orina' ${ }^{41}$.

1.2.6. Respecto a los N.A. hengur y zamankur, no tenemos constancia de ningún caso oblicuo y por tanto no sabemos si son heteróclitos $o$ temas en $-r$.

${ }^{35}$ La etimologia de esta palabra es muy discutida; Kronasser, EHS, p. 285, la relaciona con la raiz " $m \bar{e}$ 'medir' de Pokorny, $I E W$, p. 703 , lo que daría pie a reconstruir ${ }^{*} e H_{1}$; cf. Adrados, o. c., p. 107: ${ }^{*}-e H_{1}$. Sin embargo, W. Winter, "Tocharian Evidence", Evidence for Laryngeals, p. 195, prefiere postular ${ }^{*} \mathrm{H}_{3}$ y relacionar het. mehur con lat. möneo, mömentum. Este nombre, no obstante, ha dado pie a Eichner, art. cit., a formular la ahora conocida como "Ley de Eichner", por la que nombres como mehur, šehur serían temas en *-e $\mathrm{H}_{2}$.

${ }^{36}$ Bernabé, o. c., p. 576.

37 Friedrich, o. c., p. 55 s.; H. Pedersen, Hittitisch und die anderen indoeuropäischen Sprachen, Copenhague 1938, p. 187; Kronasser, EHS, p. 286.

${ }^{38}$ Bernabé, o. c., p. 385.

39 Friedrich, $H W$, p. 25; Kronasser, EHS, p. 286.

40 Bernabé, o. c., p. 573 ss.; el adjetivo panku- vendría de *penk"- "cinco', entendido como el total de los cinco dedos de la mano usados para contar, v. E. Polomé, "The Indo-European Numeral for 'five' and Hittite panku 'all"», Pratidänam. Festschrift Kuiper, 1968, pp. 98-101.

${ }_{41}$ El problema sobre el timbre de la laringal es idéntico al de mehur. 
2. El grupo de nombres heteróclitos más numeroso con diferencia lo constituyen los abstractos formados mediante sufijos compuestos de flexión heteróclita, de gran productividad a lo largo de la historia del hetita. Es una isoglosa exclusiva de esta lengua, no compartida siquiera por las restantes lenguas anatolias. Esta gran productividad de los sufijos compuestos de flexión heteróclita ha servido a Benveniste ${ }^{42}$ para reconstruir una serie de ellos: *-ter/-tn-, *-ser/-sn-, *-uer/-un-, *-mer/-mnal indoeuropeo, en donde tendrían la misma productividad que en hetita. Sin embargo, estos sufijos no se han conservado más que de forma residual en algunas lenguas, como es el caso de lat. iter *itinis o airl. arbor arbe, y en un solo caso el sufijo *-uer/-un- parece haber tenido una mínima productividad en un área dialectal restringida en el greco-indoiranio. Por ello, parece que el desarrollo de esta categoría en het., lejos de ser un arcaísmo, fue una innovación de esta lengua ${ }^{43}$.

Podemos separar dos grandes grupos: aquellos sufijos que se flexionan mediante heteróclisis $-r / n$-, esto es, el sufijo $-(a)$ tar $/-(a) n n a s ̌ ~<-(a) t-$ naš y -eššar/-ešnaš; y un sufijo en el que la alternancia es de tipo $-r / \emptyset$-:

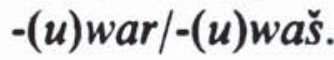

\subsection{Sufijos flexionados mediante heteróclisis $-r / n-$ :}

2.1.1. Los abstractos hetitas en -atar, en ocasiones -tar y -attar, G. -atnaš, normalmente con asimilación: -annaš, constituyen el grupo más grande de heteróclitos en $-\boldsymbol{r} / \boldsymbol{n}$-. Tienen un paradigma defectivo.

Las formas del nominativo-acusativo pueden funcionar como plurales ${ }^{4}$, aunque a veces se forma un plural en -atarri, con extensión analógica de $-r$ a toda la flexión, tipo zankilatarri. Los casos oblicuos suelen presentar asimilación -tn->-nn-, pero la forma sin asimilar se conserva en algunos casos, tipo haratni, iyatnaš, tarmatnaš. En un caso incluso aparece asimilación progresiva: huitar 'reino animal', G. huitnaš, I. huitnit, pero también G. hu-it-ta-aš. La geminada -nn- puede reducirse como en hunhuwanaš, išhaššarwanaš, karšattani, miyahundanaš, etc.

Es relativamente frecuente la grafia sin - $r$ final en el nominativoacusativo: - $t a$ o también - $d a$, interpretada por Kammenhuber ${ }^{45}$ como una tendencia del hetita a perder esta $-r$ final como resolución del paradigma heteróclito. Por su parte, E. $\mathrm{Neu}^{46}$ piensa que se trata en cada

42 Benveniste, o. c., passim.

${ }_{43}$ V. también Villar, o. c., p. 225.

4 Cf. Villar, o. c., p. 65.

${ }^{45}$ Kammenhuber, art. cit.

${ }^{46}$ E. Neu, "Hethitisch $/ \mathrm{r} /$ im Wortauslaut", Serta Indogermanica, Festschrift Neumann, 1982, pp. 205-25. 
caso de soluciones de sandhi; son los nombres alwanzata; dameda; hattata; iyada, iyata; išpata; kušduwata; miyata; paprata; pattiyata; šamanata; wagata; $S_{I} G_{7}$. $S I G_{7}$-ta. Hay una variante con $-r$ conservada en todos los ejemplos.

Por otra parte hay transferencia al género común en antuhšatar 'gente', explicado tradicionalmente como un colectivo, en el poco claro ${ }^{E Z E N}$ hahratar G. ${ }^{E Z E N h a h r a n n a s ̌, ~ y ~ e n ~ w a g a t a(r), ~ c o n ~ u n ~ N . s g . c . ~ w a g a t a s ̌ ~}$ y un A.sg.c. wagatan.

En hetita tardío tenemos constancia de un sistema de eliminación de este tipo flexivo mediante el sufijo -alli- añadido al tema en - $\boldsymbol{n}$ de los casos oblicuos: ahet. kattawatar, G. kattawannaš pasa en het. tardío a kattawann-alli-; esta palabra pasa en préstamo al luv. cuneif. kattawatnalli- ${ }^{47}$.

En general se puede aceptar la clasificación de Kammenhuber ${ }^{48}$ para los abstractos en -atar. Divide esta autora este grupo de nombres en:

- Abstractos verbales: ašatar, G. ašannaš 'asiento' sobre eš-/aš'sentarse' ${ }^{49}$.

- Denominativos: idaluwatar, D.L. idaluwanni 'maldad' de idalu-/$a u$ - 'malo' so.

- Nombres de los que no tenemos constancia de su palabra base: aštarayatar 'pecado, delito', de etimología incierta ${ }^{51}$.

Desde Benveniste ${ }^{52}$ se suele hablar de antiguos abstractos verbales en *-ter/-tn- conservados por el hetita y cuya máxima representación sería lat. iter / *itinis (itineris), het., itar, toc. A ytär, formado sobre el verbo ide. ${ }^{*} e i-/ i$ - 'ir'. Benveniste incluye algunos infinitivos: infs. apers. en -tanaiy, ai. -tari y abstractos verbales en - tn- como ai. cyautná- 'estremecimiento', av. šyaoOna- 'acción' sobre ai. cyav-, av. šyav- 'poner en movimiento'. Restos del tema en - $n$ en otras lenguas con valores diversos son los nombres lat. en -tinus: diütinus, pristinus, etc., los nombres

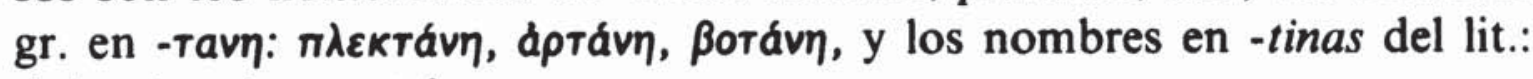
dabartinas 'presente'.

47 A. Kammenhuber, "Hethitisch, Palaisch, Luwisch und Hieroglyphenluwisch", Handbuch der Orientalistik, 1. Abt., 2. Bd., 1-2 Absch., Lief. 2, Leiden-Colonia 1969, p. 297.

${ }^{48}$ Kammenhuber, "Hethitisch...», p. 185 ss.

49 Friedrich, $H W$, Erg. 1966, p. 11; Kronasser, EHS, p. 293; Tischler, HEG,

p. 77; J. Puhvel, Hittite Etymological Dictionary (HED), La Haya 1984, p. 296.

so Kronasser, EHS, p. 295; Tischler, HEG, p. 444; Puhvel, HED, p. 490.

s1 Kronasser, EHS, p. 296; Kammenhuber, Hethitisches Wörterbuch, Heidelberg 1975, p. 491; Tischler, HEG, p. 86; Puhvel, HED, p. 219.

52 Benveniste, o. c., p. 105 ss. 
Sturtevant ${ }^{53}$ compara los abstractos hetitas en -atar con los gerundios lat. en -ndo, si aceptamos que la grafia het. - $t$ - aquí representa /d/ y que en lat. se ha producido una metátesis $-d n->-n d-$.

Carecemos de nombres de agente en -ter, categoría que ha sido reemplazada por los nombres en -talla-, excepto ekuttara- 'escanciador' y weštara- 'pastor'. Benveniste ${ }^{54}$ interpreta esta carencia relacionando directamente los nombres en -tar/-nnaš, según él de sentido intransitivo o medio, y los nombres en *-ter, cuyo sentido sería "el de un adjetivo que marca el ejercicio de una actividad no transitiva». Asimismo incluye aquí los nombres de instrumento en *-tro, en los que «la tematización produce una individualización de la noción». Sin embargo, encontramos múltiples ejemplos de deverbativos formados sobre raices transitivas, del tipo karšatar 'sección de un campo, castración' sobre el verbo karš- 'cortar', kunatar 'homicidio' sobre kuen- / kun- 'matar', tarmatar 'atadura' sobre tarma- 'atar', etc ${ }^{55}$.

E. Laroche ${ }^{56}$ se apoya en esta distinción para postular la existencia de un sufijo primario de nombres de acción - $t$ - que aparece en los nombres de actor en *-tör y de agente en *-ter y en hetita en los abstractos en -atar y también en una serie de nombres deverbativos en - $(a) t$ - de género inanimado, tipo N.sg. aniyaz, A.sg. aniyatan, G.sg. aniyataš 'operación, acción', sobre aniya- 'hacer un trabajo'. Parece, por tanto, que el alargamiento $-t$ que aparece con un valor puramente fonético en otras lenguas: ai. yákr-t, gr. ந̆na-T-os, se ha morfologizado en indoeuropeo para marcar nombres de acción. Otras lenguas conservan también abstractos sobre tema en $-t$, alargado mediante ${ }^{*}-e H_{2} /-H_{2}:$ gr. $\beta 10-T-\eta$, lit. gyva-t-à, lat. uita ${ }^{57}$.

La matización de Laroche da pie a hacer una clasificación fundamental dentro del sufijo -atar, -tar en hetita.

Tendremos por un lado un grupo de abstractos en -atar, el más numeroso, en los que *-ter/-tn- constituye una solución fonética para los temas en *-e $\mathrm{H}_{2} /-\mathrm{H}_{2}$ que no se integraron bien en el sistema flexivo del hetita. Este tipo es exclusivo de esta lengua.

53 Sturtevant, "Hittite Verbal Nouns in -tar and the Latin Gerund», Language 20, 1944, pp. 206-11; v. R. Thurneysen, "Urspr. dn tn $c n$ im Lateinischen", $K Z 26$, 1883, pp. 301-14; P. P. Persson, De origini ac ui primigenia gerundii et gerundiui latini, Upsala 1900.

$\$ 4$ Benveniste, o. c., pp. 108-10; Hittite et indo-européen, París 1962, p. 95 ss.

ss V. esta misma opinión en Sturtevant, o. c., p. 72.

s6 E. Laroche, «Noms d'action en indo-européen d'Anatolie», Mélanges linguistiques offerts à E. Benveniste, Lovaina 1975, pp. 339-46.

${ }^{37}$ F. Specht, Der Ursprung der indogermanischen Deklination, Gotinga 1944, p. 346 . 
Que los abstractos het. en -atar sean temas en ${ }^{*} \mathrm{H}_{2}$ lo prueba la relación con los abstractos luvitas en -ahi( $t$, puesta de relieve por $\mathrm{C}$. Watkins y A. Bernabé ${ }^{58}$ : son los nombres luv. annarum $(m)$ ahi $(t)$, het. innarahhuatar; luv. adduwalahi( $(t)$, het. idaluwatar; luv. hattulahi( $(t)$, het. hatdulatar; luv. huitwalahi( $t)$, het. huišwatar; luv. nakkǔšsahi( $t)$, het. nakkušatar. Las formas luv. en -abi, alargadas con una - $t$ secundaria


* $e \mathrm{H}_{2}$ en grado pleno, son las mismas que dan lugar a los abstractos he-


teróclitos en $-r / n-59$.

Por otro lado hay un grupo más antiguo de abstractos en -tar/-nnaš que son herederos directos del sufijo ide. *-ter/-tn-, que, a su vez, es analizable como la resolución mediante heteróclisis del sufijo primario *-t- de nombres de acción. Este grupo es muy reducido: serán los deverbativos galaktar, huitar ${ }^{60}$, itar (cf. también lat. iter ${ }^{*}$ itinis, toc. A ytär), kallištar y los nombres de declinación especial kuttar kuttanaš (deverbativo sobre la raiz ide. " $g \bar{e} u-g \bar{u}$ ) y uttar uddanaš (deverbativo sobre la raiz ide. "wek"-); y dos nombres de los que desconocemos su palabra base: kuptar y ${ }^{516}$ puttar $^{61}$.

2.1.2. Los abstractos en -ššar, -eššar son un grupo menos numeroso que los en -atar, pero menos defectivo que éstos, dado que tienen una declinación muy desarrollada en singular y algunos casos de plural ${ }^{62}$.

El nominativo-acusativo de singular suele aparecer en la forma -eššar con la variante $-\check{s}^{2}{ }^{63}$. La geminada -šš- probablemente procede de una asimilación del grupo ${ }^{*}$-ešr ${ }^{64}$. Si admitimos con Sturtevant ${ }^{65}$ que

${ }^{58} \mathrm{C}$. Watkins, «Die Vertretung der Laryngale in gewissen morphologischen Kategorien in den indogermanischen Sprachen Anatoliens", Flexion und Wortbildung, ed. H. Rix, 1975, pp. 358-78, y A. Bernabé, "The Luwian abstracts in -ahi( $t)$ and the Hittite nouns in -ai-. A critical Review", Linguistic Happening. Hom. B. Schwartz, Lovaina 1988, pp. 105-27, quien reconstruye ${ }^{*}-\mathrm{eH} i$.

$59 \mathrm{~V}$. Kammenhuber, art. cit.

${ }^{\infty}$ Prueba de que huitar no es un tema en ${ }^{*}-H_{2}$ es que se corresponde con un nombre luv. en -mar: huitumar, y no con un tema en -ahi( $t)$.

${ }^{61}$ El denominativo $d / \operatorname{tam}(m)$ etar 'abundancia', que en principio podria ser incluido en este grupo, puede ser un tema en ${ }^{*} H_{2}$ de resultado - $\bar{e}$ - según la llamada ley de Eichner, como postulan A. Morpurgo Davies - J. D. Hawkins, "The late hieroglyphic Luwian Corpus; some new lexical Recognitions", Hethitica 8, Homm. Laroche, ed. Lebrun, 1987, pp. 267-95.

62 Kammenhuber, "Hethitisch...", p. 187.

${ }^{63}$ Cf. Kronasser, EHS, p. 288. 415-56.

4 A. Bernabé, "Geminación de $-s$ y sonantes en hetita», RSEL 3, 1973, pp. 1933.

${ }_{65}$ Sturtevant, A Comparative Grammar of the Hittite Language, Philadelphia 
son temas en $-s$ flexionados mediante heteróclisis, la presencia o ausencia de $-e$ en el N.A.sg. respondería a una alternancia grado pleno/grado cero tardia, ya no motivada.

El genitivo es en -ešnaš, el dativo en -ešni, -ešna, el ablativo en -ešnaz(a), -ešnanza y el instrumental en -ešnit. Como N.A.pl. suele funcionar la forma de singular, excepto en los casos zankilatarri ${ }^{H ! A}$, šuppešša$r i^{H I . A}$ y uppeššarritl.A, en donde se ha extendido analógicamente la $-r$ del N.A.sg.

Igualmente parece haberse extendido la $-r$ del nominativo por analogía en el I. išpantuzzeššarit.

Son también relativamente frecuentes los N.A. sin -r (hannešša, hattešša $(r)$, hatrešša, išhešša, išhuzzi(y)ašša $a(r)$, luešša, paprešša, šarlamišša, wakišša) ${ }^{66}$.

En cuanto al G. hunhuwanaš, atribuido a hunhueššar, parece más plausible la explicación de Kronasser ${ }^{67}$ : se trata del genitivo de un tema en -atar cuyo N.A. *hunhuwatar no está atestiguado.

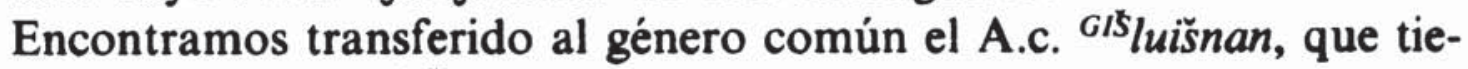
ne al lado un N.A.n. ${ }^{G 1 \zeta}$ lueššar.

Al igual que los nombres en -atar, el sufijo -eššar puede crear:

- Abstractos verbales, como karšeššar, G. karšešnaš, D.L. karšešni 'corte' sobre el verbo karš- 'cortar, separar' ${ }^{68}$.

- Denominativos, a partir de sustantivos y adjetivos: parkuě̌šar, G. parkuešnaš 'limpieza' de parkui- 'limpio' ${ }^{69}$.

- Nombres de los que no tenemos constancia de su palabra base:

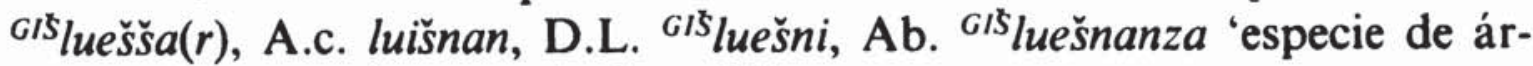
bol o planta usada como incienso' ${ }^{70}$.

En las otras lenguas anatolias no hay nada parecido a este sufijo, contando con el problema de dispersión en la que se nos presenta su material. El luvita cuneiforme testimonia el nombre temático (UZU)happiša comparable al het. (UZU)happeššar; el luv. jerogl. muestra un sufijo temático -ašara- en el D. kutašari, que será igual que el het. *kuttešni de kutteššar. Aparece con otro sufijo la palabra cultural de origen extranjero luv. jerogl. tunakalaš, comparable al het. tunakeššar ${ }^{71}$.

66 Neu, art. cit., p. 208.

67 Kronasser, EHS, p. 290.

68 Tischler, $H E G$, p. 520.

${ }^{69}$ Kronasser, $E H S$, pp. 290, 294.

70 Friedrich, $H W$, Erg. 1966, p. 22 s.; Kronasser, $E H S$, p. 29; Güterbock-Hoffner, The Hittite Dictionary..., p. 73.

7 Kammenhuber, "Hethitisch...», p. 187; Kronasser, EHS, p. 321. 
Sturtevant ${ }^{72}$ pensó en un primer momento que esta flexión no tenía paralelo en las restantes lenguas ides. y postuló que los temas en -eššar eran el tratamiento mediante heteróclisis de los temas ides. en $-s$.

En apoyo de la tesis de Sturtevant de que estos nombres en -ě̌šar son temas en $-s$ flexionados mediante heteróclisis está el hecho de que hay en otras lenguas temas en $-s$ que forman abstractos a partir de ad-

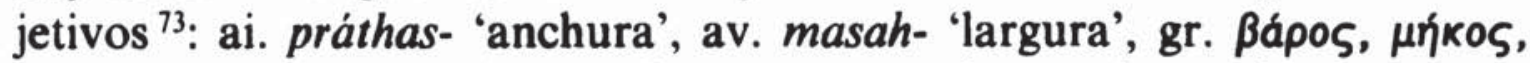
пáxos, крáтos, lat. albor, rubor, etc., o sobre verbos: ai. vácas-, gr. Ennos; ai. ápas-, lat. opus, etc. Además, si no es en los temas en -eššar, los temas ides. en *-es/-os no aparecen en ningún tipo productivo en hetita, ya que los temas het. en -ě̌ (-ǐ̌) y en -aš son poquísimos.

Sin embargo, Benveniste ${ }^{74}$ encuentra restos de este sufijo en las formaciones temáticas en ${ }^{*}$-sno- y los femeninos en ${ }^{*}$-snā: ai. krt-sná'completo', tikk-ṣna- 'agudo', mrit-sná- 'polvo', lat. arānea, gr. doaxví $<{ }^{*}$ arak-sn[ä]; lat. cèna, alat. cesna (Fest.), osc. kersnu 'cena' < *kerstsnä, etc. También los infinitivos ai. en -sani, ya estén formados sobre la raíz bhüșaṇi, ya sobre el tema de presente tarișaṇi. Relacionados con estos infinitivos estarán igualmente los infinitivos gr. en - $\varepsilon$ iv si es que proceden de *-e-sen.

Después del trabajo de Benveniste, Sturtevant rectifica su anterior hipótesis ${ }^{75}$ y sigue la propuesta de Benveniste. Sin embargo, hasta tal punto parece claro que estos casos son alargamientos en $-n$ sobre temas en $-s$ que el propio Benveniste así lo reconoce: ai. vakșána-(i), tema alargado por -s: vak-ș-, cf. gr. aú̧ávw; lat. lüna < *leuk-s-na, aunque él mismo dice que éstos son "pequeños accidentes». Es de notar que en las lenguas sólo se han conservado formaciones sobre tema en - $n$ : -sen, $-s n-o,-s n-\bar{a}$, pero nunca sobre el tema en $-r$, a no ser airl. när 'modesto', si es que procede de ${ }^{*} n \bar{a}-s r o-{ }^{76}$.

2.1.3. Hay una serie de nombres en los que se puede analizar un sufijo -war / -unaš, heredero del sufijo ide. "wer/-un- descrito por Benveniste ${ }^{77}$; estos nombres son comparables a los nombres av. en -var-/-vany a los griegos en -Fap/-Fatos. Son pocos, pero se pueden identificar dos funciones en este sufijo: puede crear designaciones de partes del cuerpo (animal): karawar 'cuerno' y partawar 'ala' y crea también deverbativos: ašawar 'aprisco', aššiyawar 'amor' y haršawar 'agricultura'.

72 Sturtevant, A Comparative Grammar..., 1933.

V. Specht, o. c., p. 346.

Benveniste, o. c., p. 101.

V. Sturtevant, A Comparative Grammar..., 1964, p. 24.

7 Sturtevant, Indo-Hittite Laryngeals, Baltimore 1942, p. 24.

77 Benveniste, o. c., pp. 110-20. 
Estos nombres en -war/-unaš se suelen interpretar como el grado pleno ${ }^{*}$-or correspondiente a un grado cero que aparece en los nombres en -ur/-unaš, tipo mehur, G. mehunaš ${ }^{78}$. Sin embargo, si admitimos que los nombres en -ur/-unaš son grados cero en los que *-r $>$-ur condicionada por la presencia de laringal (mehur) o labiovelar (pankur), habrá que admitir también que los nombres en -war/-unaš responden a otro origen distinto y que constituyen un sufijo autónomo.

2.1.3.1. N.A. ašawar, D.L. ašauni, het. rec. ašaunišši, Ab. ašaunaz 'aprisco'; relacionado con el verbo eš-/aš- 'estar, encontrarse, sentarse' ${ }^{79}$.

2.1.3.2. N.A. aššiyawar, I. aššiyaunit 'amor', relacionado con el verbo aššiya- 'querer' ${ }^{80}$.

2.1.3.3. N.A. haršawar, G. haršaunaš 'agricultura, acción de arar' está relacionado con el verbo harš- 'arar' ${ }^{81}$. Cabe reconstruir una raiz ${ }^{*} \mathrm{H}_{2}$ er- con un alargamiento $-s$ posterior relacionable con gr. a $\rho \delta \omega$, lat. arō y sobre todo con el heteróclito airl. arbor G. arbe, nombre en *-wer/-un- sobre esta misma raíz verbal.

2.1.3.4. Eichner ${ }^{82}$ incluye dentro de este tipo el N.A. karawar 'cuerno, asta' por paralelismo con los anteriores, aunque carece de casos oblicuos documentados para saber si se trata de una formación heteróclita: puede ser un tema -war/-waš, como piensa Friedrich ${ }^{83}$. La comparación con otras formaciones heteróclitas de la misma familia como gr. кápa крáatos, ai. śíras sìrș̣ás hace más verosímil la hipótesis. Sin embargo, Nussbaum ${ }^{84}$ localiza el L. garauni que garantiza la inclusión de karawar en este grupo.

2.1.3.5. N.A. partawa(r), G. partaunaš, I. partaunit 'ala' ${ }^{85}$.

${ }_{78} \mathrm{~V}$. Eichner, art. cit.

${ }^{79}$ Kronasser, $E H S$, p. 298; Puhvel, $H E D$, p. 296. Kammenhuber, $H W$, p. 393 , no obstante, opina que es de etimología desconocida.

${ }_{80}$ Kronasser, $E H S$, p. 293; Kammenhuber, $H W$, p. 403; Tischler, $H E G$, p. 83; Puhvel, $H E D$, p. 191.

${ }^{81}$ Kronasser, $E H S$, p. 298; Eichner art. cit.; Tischler, $H E G$, p. 182.

${ }^{82}$ Eichner, art. cit.

${ }^{83}$ Friedrich, $H W$, Erg. 1955, p. 102.

${ }^{84}$ Nussbaum, o. c., pp. 32, 46. Más difícil es saber si la forma SI-ar, I. SI.HI.anta $(\mathrm{H}$. Ehelof, "Zu dem Instrumentalis auf $-t$ im Hethitischen», IF 43, 1926, p. 317) pertenece a este grupo de palabras. H. Frisk, Kleine Schriften zur Indogermanistik und zur griechischen Wortkunde, Göteborg 1966, p. 26, asi lo opina, y reconstruye *kerar *kerant $(a)$.

${ }_{85}$ Kronasser, $E H S$, p. 283 lo relaciona con aesl. pero 'pluma', parit 'volar', ai. parna 'pluma', aunque tampoco ve clara esta formación. Es posible que esta palabra proceda de la raiz * pter- que da lugar al heteróclito pattar pattanaš con metátesis de $-r$ y sufijación en -war. 
2.2. Hay que hacer un apartado especial para el grupo más numeroso de sustantivos verbales, que están formados mediante un sufijo que presenta una alternancia especial, no testimoniada en ninguna otra lengua ide.: $-r / \phi$ - Es muy llamativo que estos temas no presenten $-\boldsymbol{n}$ en los casos oblicuos, por lo que Villar ${ }^{86}$ habla de un nuevo tipo de heteróclisis en el que se opone un nominativo-acusativo con alargamiento en $-r$ al resto de los casos en los que las desinencias se unen directamente al tema en $-u$.

El nominativo-acusativo es en -war. En ocasiones el sufijo se reduplica, tipo wahnuwawar (que alterna con wahnuwar) sin que se pueda explicar como un fenómeno gráfico ni se pueda dar una regla segura. El genitivo correspondiente a estos temas es en -waš, que es idéntico al de los temas en $-u^{87}$ del tipo aššuwaš 'bueno'.

Es un tipo extraordinariamente defectivo, incluso es frecuente la ausencia de testimonio del nominativo-acusativo correspondiente al genitivo en -waš. Los únicos casos oblicuos distintos del genitivo son los ablativos armahhuwazza, haneššuwaz y kardimiyawaz, formados también, como el genitivo, igual que los de los temas en $-u$.

Es de notar que en las secuencias $u-w$ se produce una disimilación en -um- dando lugar a formas de tipo arnumar, G. arnumaš. Es un fenómeno fonético, y no gráfico como pretende Kronasser ${ }^{88}$; tiene excepciones, al igual que las tiene la asimilación $-t n->-n n-$. Las grafias vacilan entre $-m-$ y $-m m-$.

Tenemos algunos casos de extensión analógica de $-r$ a los casos oblicuos en el N.A.pl. guršawara de guršawar; N.A.pl. halhaltumari de halhaltumar; N.A.pl. minumarri de minumar, G. minummaš, minuwaš; Ab. šallanumarraza de *šallanumar; D.L. šawarri de šawar; N.A.pl. tahdumara de tahdumar y Ab. wekuwarraz de wekuwar.

Existen algunos casos oblicuos problemáticos que presentan - $n$ : guršawar, Ab. guršawanaza; huitiyawar, G. huitiyawaš pero D.L. huittiyau$n i$; išiyahhuwar, D.L. išiyahhuwanni; iyawar, G. iyawaš, D. iyawanna; wehuwar, G. wehuwaš, pero existe también un G. we/ahannaš. Creo que en estos casos nos encontramos con una introducción secundaria de $-n$ analógica de la - $n$ - de los temas de los casos oblicuos de los otros sufijos que forman abstractos. Mediante este proceso se produce una nivelación entre el sufijo -war/-waš y el sufijo -war/-unaš, menos productivo, pero también capaz de formar abstractos.

\footnotetext{
s6 Villar, o. c., p. 92.

${ }^{87}$ Kammenhuber, art. cit.; Kronasser, EHS, p. 299.

${ }^{88}$ Kronasser, $E H S$, p. 81 ss.
} 
Los nominativos-acusativos sin $-r$ son mucho menos frecuentes que en los otros sufijos heteróclitos, estando limitados a tarnuma y quizá también iyawa ${ }^{89}$, en cambio hay constancia de genitivos $\sin -\check{s}$ final: nuntarnuwa G. de nuntarnuwar; šarikuwa G. de šarikuwar y warnuwa G. de warnuwar.

Podemos clasificar los nombres en -war/-waš en tres grandes grupos:

- Abstractos verbales, como arkuwar, G. arkuwaš, N.A.pl. arku(w)arri 'súplica, ruego' de arkuwai- 'rogar' 90.

- Denominativos. Son pocos, pero existen, por ejemplo halluwawar 'profundidad' en relación con hallu- 'profundo' 91 .

- Nombres de los que no tenemos constancia de su palabra base, como puntaryawar (?) ${ }^{92}$.

En el citado trabajo de Benveniste se describian los sufijos de flexión heteróclita *-ter/-tn-, *-ser/-sn-, *-mer/-mn- y *-uer/-un-. Sin embargo los propios estudiosos coetáneos de Benveniste podían comprobar que en het. no habia restos tan abundantes del sufijo *-uer-/-un- como había supuesto Benveniste. Esto provocó que algunos indoeuropeístas identificasen el sufijo het. -war/-waš como la evolución histórica del sufijo *-uer/-un-. Naturalmente esto comporta ciertas dificultades fonéticas y morfológicas. H. Pedersen ${ }^{93}$ explica los genitivos en -waš como procedentes del protoanatolio *-wanš. Unánimemente se analiza el infinitivo luv. en -una como un caso oblicuo de estos temas, de modo que se reconstruye sumativamente un sufijo ${ }^{*}$-uer/-un- ${ }^{94}$. Parece forzoso puntualizar que los infinitivos (o supinos, según los autores) en -una no se encuentran en hetita, sino en luv. cuneif. aduna, luv. jerogl. aruna y pal. ahuna. En cambio, el het. presenta infinitivos en -anna, del tipo adanna y akuwanna, que parecen estar relacionados con los abstractos en -atar/-annašs. Estarán efectivamente relacionados con el tema en - $n$ del sufijo alternante *-uer/-un- los infinitivos het. en -uwanzi y los supinos en -uwan ${ }^{95}$.

Otra solución apuntada para explicar esta flexión tan anómala es la de Benveniste ${ }^{96}$ : este grupo de abstractos verbales (infinitivos, en su de-

${ }^{89}$ V. Neu, art. cit., p. 221.

${ }^{90}$ Kronasser, $E H S$, p. 301; Tischler, $H E G$, p. 61.

91 Kronasser, $E H S$, p. 306; Tischler, $H E G$, p. 138.

${ }^{92}$ Kronasser, EHS, p. 307.

93 H. Pedersen, 0 . c., p. 45; K. Hoffmann en Eichner, art. cit., p. 92.

${ }^{4}$ Benveniste, o. c., p. 113; Sturtevant, o. c., p. 74; Kronasser, EHS, p. 299; Kammenhuber, art. cit., "Hethitisch...", p. 262.

${ }_{95}$ A. Kammenhuber, "Studien zum hethitischen Infinitivsystem, I. Die Morphologie der Infinitive auf -anna (-atar) und -uwanzi (-uwar)", MIO 2, 1954, pp. 44-77.

\%6 Benveniste, o. c., p. 120. 
finición) son temas en $-u$ que han tomado $-r$ en el N.A. secundariamente a partir de los numerosos abstractos y neutros que estaban caracterizados por esta marca.

Por otra parte, pensamos que en *-r quizá pueda reconstruirse un valor de formador de abstractos, que podría explicar esta peculiar formación hetita; cf. los abstractos toc. en $-r$ del tipo: toc. A kursär B kursar 'lengua, vehículo', A kuryar B karyor 'comercio', AB pikär 'gesto', B Ab. sg. kakämarmem, Perlat. sg. kakämarsa, y adverbios del tipo A șkara B aṣkar 'detrás' 97 .

2.3. Un problema sobre el que se debaten todas las gramáticas hetitas es el de si se puede describir un sufijo -mar autónomo. Como ya hemos visto, la secuencia - $u$-war se puede disimilar en -u-mar y así por ejemplo Sturtevant ${ }^{98}$ considera que todos los nombres en -mar son simples variantes de los en -war. Sin embargo, Benveniste ${ }^{99}$ postula la existencia de un sufijo ide. autónomo *-mer/-mn- que se conservaría en hetita en nombres como tarnumar, y residualmente en nombres como gr. $\lambda \tilde{u} \mu a \rho$, en los infinitivos gr. en - $\mu \varepsilon v$, - $\mu \varepsilon v a \iota$ e i.-ir. en *-manai, y en los nombres en *-men que se conservan en todas las lenguas ides.

Kronasser ${ }^{100}$ mantiene la hipótesis de un sufijo autónomo het. -mar/-mnaš y se apoya en el testimonio de instrumentales del tipo miumnit, šaramnit. En cambio Kammenhuber ${ }^{101}$ considera que los casos del sufijo -mar son demasiado escasos y demasiado oscuros como para garantizarnos la existencia de un sufijo productivo. Laroche ${ }^{102}$ opina, con muy buen criterio, que dicho formante carece de valor semántico clasificatorio como para poder hablar de sufijo.

Efectivamente, sigue Laroche, hay unos pocos nombres en -mar que no son producto de ninguna disimilación y que en realidad son nombres en -ar/-anaš (como pueda serlo mukar) sobre un alargamiento - $m$ de la raiz.

2.3.1. "halwamar, Ab. haluamnaz 'celo, aplicación' ${ }^{103}$.

97 Cf. A. J. Van Windekens, Le tokharien confronté avec les autres langues indoeuropéennes, Lovaina 1979, pp. 66-71.

98 Sturtevant, o. c., p. 74.

99 Benveniste, o. c., pp. 116-8.

100 Kronasser, EHS, p. 319.

101 Kammenhuber, art. cit.

102 Laroche, "Etudes de vocabulaire, VI», $R H A$ 15, 1957, pp. 9-29.

${ }_{103}$ Kronasser, $E H S$, p. 320; Tischler, $H E G$, p. 138. 
2.3.2. El confuso hilammar, kilammar, G. hilamnaš, hilannaš, D.L. hilamni kilamni hilamna 'patio, pórtico, propileo' ${ }^{104}$.

2.3.3. miumar, I. miumnit 'benevolencia' ${ }^{105}$.

2.3.4. šaramar, I. šaramnit ${ }^{106}$.

Por otra parte tenemos otros nombres en -mar sin caso oblicuo documentado y que no podemos adscribir a ningún tema con seguridad; en ellos es frecuente la ausencia de $-r$ final en el nominativo-acusativo, que es el único caso atestiguado: alpuemar, alpuema; arpamar 'nombre de un pan funerario'; harnamar, harnama 'levadura'; immimar 'mezcla'; tiyamar, tiyama '¿cuerda?'.

2.4. Otro aspecto debatido por las gramáticas es la oposición de significados entre los diferentes abstractos verbales que se crean a partir de los tres sufijos en -atar, -eššar y - $(u)$ war.

La communis opinio, a partir de A. Götze ${ }^{107}$ es que los nombres en -eššar son nomina actionis de un significado más concreto que los nombres en -atar, y pone por ejemplo ištapeššar 'esclusa' sobre ištap(p)- 'cerrar'. Por su parte, Kronasser ${ }^{108}$ piensa que los nombres de -uwar son exclusivamente deverbativos.

De todos modos, el trabajo más completo al respecto es el de Kammenhuber ${ }^{109}$. Sus puntos de vista son los siguientes:

En ningún caso son intercambiables los tres sufijos; cada uno de ellos tiene un valor concreto.

Los nombres en -eššar crean abstractos que señalan el efecto de la acción verbal y son más concretos que los restantes; el sufijo -eššar forma sólo nombres de acción. Cuando -eššar crea abstractos a partir de adjetivos lo hace sólo a partir de temas en $-i$, aunque secundariamente puede formar abstractos a partir de un tema en - $u$ : dicha extensión parte de la interinfluencia entre los adjetivos parku- 'alto' y palhi- 'ancho'; por ello se crea el derivado parkueššar, sinónimo de parkuwatar.

104 Laroche, art. cit., reconstruye *hilatar para explicar el G. hilannaš, forma que habitualmente se explica como asimilación regresiva a partir de hilamnaš (Friedrich, $H W$, Erg. 1957, p. 6; Tischler, HEG pp. 243 s., 570). Según Kammenhuber, art. cit., sólo se puede hacer constar que esta palabra pertenece de alguna manera a hila- "patio'. Es también posible que sea un préstamo del asirio.

105 Kronasser, EHS, p. 319.

106 Kronasser, EHS, p. 320.

107 A Götze, Madduwattaš, Mitteilungen der vorderasiatischen-aegyptischen Gesellschaft 32, 1, 1928, p. $62 \mathrm{~s}$.

${ }_{108}$ Kronasser, EHS, pp. 301-8.

109 Kammenhuber. "Studien zum hethitischen Infinitivsystem, III. Die deverbalen und denominalen -atar- und -eššar Abstracta», MIO 2, 1954, pp. 403-44. 
Los nombres en -eššar muestran una especialización en la capacidad que tienen para adaptar palabras culturales de origen extranjero: tunnakeššar, tuhhueššar.

Los nombres en -atar están más próximos al significado básico del verbo, aunque hay casos en los que se produce una transferencia secundaria del significado del verbo: hattatar 'sabiduria' (si es que es deverbativo de hatta- 'rasgar, perforar') / hatteššar 'hoyo, agujero'.

El sufijo -atar forma sustantivos verbales, correlatos de los infinitivos en -anna, y abstractos verbales. Forma también denominativos a partir de todo tipo de adjetivos. Cuando se añade a un tema en - $i$ toma un significado más concreto, que lo define como formación nueva, frente al derivado en -eššar: šuppiyatar 'parte luminosa de la luna creciente' / šuppeššar 'purificación'. Los denominativos en -atar se especializan en la creación de derivados a partir de nombres de parentesco: anniyatar 'maternidad'.

El sufijo - (u)war forma sólo sustantivos verbales, correlatos de los infinitivos en -uwanzi.

No obstante, creo que se debe matizar alguna de estas afirmaciones, ya que donde se mostrará una auténtica oposición de significados es en los casos en los que una misma raíz da lugar a varios derivados, hecho que es muy frecuente.

En estos casos hay una cierta tendencia a que los nombres en -atar asuman significados más concretos frente a los nombres en -eššar: hannešnatar 'sentencia'/ hanneššar 'causa, pleito, derecho', de hanna- 'fijar, regular'; papratar 'mancha' / papreššar 'impureza', de paprahh- 'hacer impurezas'. En cambio en otros casos son perfectamente sinónimos: palhatar/palheššar 'anchura', de palhi- 'ancho'; pargatar / parkeššar, 'altura', de parku- 'alto'; precisamente en los casos en los que Kammenhuber descubre una transferencia secundaria de significado: šupiyatar/ šuppeššar, el nombre en -atar es marcado respecto al nombre en -eššar.

Por otra parte, tampoco hay oposición clara entre los nombres en -war y los nombres en -atar en casos como armahatar/armahhuwar 'embarazo' de armah- 'embarazar'; innarahhuar/innarawatar 'fuerza', de innarah(h)- 'obrar vigorosamente'; y cuando hay diferencia de significados parece resultado de concreciones diferentes: karšatar 'sección de un campo, castración' / karšuwar 'un funcionario', de karš- 'cortar'; kunnahhuwar 'hecho correcto' / kunnatar 'rectitud en el oráculo', de kunna'bien', aunque nuevamente el nombre en -atar parece algo más concreto.

Hay que hacer notar que - $(u)$ war puede formar también abstractos sobre adjetivos: halluwawar y sobre participios: pittuliyawar. Es un uso 
poco frecuente y muy posiblemente extendido analógicamente a partir de su función como creador de sustantivos verbales, pero no por ello debe ser olvidado.

Los nombres en -eššar y los nombres en -war son perfectamente sinónimos en numerosas ocasiones: arkueššar/arkuwar 'oración', de arkuwai- 'orar'; išheššar/išhiyawar 'atadura' de išhai- 'atar'; walheššar/walhuwar 'ataque', de walh- 'atacar', etc. Cuando hay oposición de significados son más concretos los nombres en -war: išhuwawar 'escombros'/ išhueššar 'carga, montón, simiente'; parheššar 'prisa'/ parhuwar 'galope', de parh- 'apresurarse'; tarupeššar 'totalidad'/ taruppuwar 'reunión', de tarup- 'reunir'; wahnueššar 'vuelta'/ wahnumar 'vallado', de wahnu- 'darse la vuelta'.

En definitiva, parece que nos encontramos ante sistemas todavía no dotados de la suficiente caracterización como para poder hablar de oposiciones regulares entre ellos. Poco a poco se van polarizando las oposiciones de significado + concreto / - concreto, pero en el corte sincrónico en el que encontramos la lengua hetita éstas no están todavía perfectamente establecidas. Los nombres en -atar tienden a ser más concretos que los nombres en -war y éstos más concretos que los nombres en -eššar, que parecen tener un significado más general.

En un esquema podemos representar los casos en los que hay oposición de significados, representando $(+)$ la marca de + concreto:

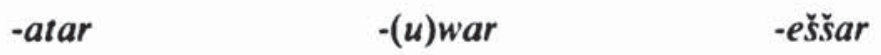

Se oponen a

-atar

$-(u)$ war

-eššar

\begin{tabular}{|c|c|c|}
\hline- & kunnahhuwar (-) & hanneššar (-) \\
\hline kunnatar (+) & - & parheššar (-) \\
\hline hannešnatar (+) & parkuwar (+) & - \\
\hline
\end{tabular}

3. Conclusiones.

El hetita presenta dos tipos distintos de heteróclisis.

El tipo $-r / n$ - que es heredado y relativamente productivo forma nombres dentro de campos semánticos bien delimitados: designaciones temporales: mehur mehunaš, partes del cuerpo: kuttar kuttanaš, fluidos: watar wetenaš y elementos básicos de la Naturaleza: pahhur pahhuenaš.

El tipo - $r / \phi$ - aparece sólo en el sufijo -war/-waš que forma abstractos del tipo taruppuwar taruppuwaš 'reunión'. Es un desarrollo exclusivo del hetita. 
Hay tres sufijos que se flexionan mediante alternancia -r/n-: -(a)tar / -(a)nnaš < -(a)tnaš; -eššar/-ešnaš; -war/-unaš.

El desarrollo enorme de estos sufijos, que es un rasgo exclusivo del hetita, parece una innovación propia de esta lengua destinada a integrar dentro de su flexión determinados temas.

El sufijo -tar/-nnaš, que aparece en unos pocos nombres y que debe de ser el heredero de un sufijo ide. *-ter/-tn-, es reutilizado para integrar los temas en ${ }^{*}-e H_{2} /-H_{2}$ que ya desde el ide. formaban abstractos y que no presentan ningún otro tipo productivo en hetita. Éste sería el origen del sufijo -atar/-annašs.

El sufijo -eššar/-ešnaš integra en el sistema nominal het. los nombres ides. en *-es/-os que ya en ide. formaban abstractos y que no aparece en ningún otro tipo flexivo hetita.

El sufijo -war/-unaš, en cambio, no es productivo y puede crear designaciones de partes del cuerpo de los animales y abstractos.

Juan Antonio Álvarez-Pedrosa 